\title{
Engineering Terminal Disulfide Bonds Into DNA
}

Characterization of synthetic oligonucleotides shapes much of the understanding of "native," higher-molecular-weight DNA and RNA molecules. Although of immense utility, short oligonucleotides usually possess lower structural and thermal stability and have greater end effects than the larger nucleic acid constructs they are intended to model. Hence, the physiochemical properties of oligonucleotides may not always compare favorably to those of larger nucleic acids (Elson et al., 1970; Scheffler et al., 1970; Baldwin, 1971; Record and Lohman, 1978; Breslauer, 1986; Breslauer et al., 1986; Olmsted et al., 1991; MacGregor, 1996; SantaLucia et al., 1996). Arguably, the most successful approach to stabilize oligonucleotides is to connect the strands that comprise a helical structure with an interstrand cross-link (Pinto and Lippard, 1985; Borowy-Borowski et al., 1990; Kirchner and Hopkins, 1991; Boger et al., 1991; Sigurdsson et al., 1993; Willis et al., 1993). Methods to cross-link nucleic acids can generally be divided into two catagories. In the first category, cross-links are formed using an exogenous reagent, most commonly a (bis)electrophile. Because (bis)electrophiles can react with nearly all of the nucleophilic sites on the bases, these agents often have little or no sequence specificity and form complex mixtures of cross-linked aducts (Millard et al., 1990, 1991; Kirchner et al., 1992). Although some natural products (like mitomycin C) and synthetic compounds (such as cisplatin and psoralen) can form lesions at unique sites, these reagents require the presence of specific recognition sites within a target sequence to generate the cross-link, which limits their utility (Sherman et al., 1985; Tomasz et al., 1987; Teng et al., 1989; Lemaire et al., 1991). In some cases, cross-link formation by alkylating agents can also have undesirable effects such as disrupting base stacking (Sherman et al., 1985; Millard et al., 1990).

In the second category, cross-links can be introduced into oligonucleotides through solidphase synthesis of oligomers site-specifically labeled with modified nucleosides that present reactive groups. Positioning the reactive groups in proximity on opposing strands of a helix allows for the formation of a cross-link. This general strategy requires appropriate selection to form the cross-link. In one of the most effective examples of this approach, Webb and Matteucci (1986) demonstrated that DNA oligomers containing cytosines bearing an aziridine group on the N4 position form cross-links after annealing to a complementary oligomer, followed by selective opening of the aziridine by the exocyclic amine of an opposing $\mathrm{dC}$ or dA. These cross-links have proved useful for some experiments, but this chemistry in particular, and related methods in general, have several limitations. For example, ethanobridged cross-links are not formed in high yield, the internal mismatch required to form the cross-link can give rise to (local) disruption of helical geometry, and the resulting crosslinks can potentially interfere with protein and drug binding (Catalano and Benkovic, 1989; Cowart et al., 1989; Cowart and Benkovic, 1991).

An alternate approach involves incorporating an intrastrand cross-link at the terminus of a helical structure. For example, one end of a duplex can be covalently linked by bridging the 3 '-and 5'-terminal hydroxyl groups with either oligonucleotide, oligoglycol, or alkyl linkers to form stem-loop structures or "hairpins" (Durand et al., 1990; Kool, 1991; Rumney and Kool, 1992; Salunkhe et al., 1992; Bannwarth et al., 1994; Gao et al., 1994, 1995; Williams and Hall, 1996). Both ends of a duplex can also be covalently capped with linkers to generate double hairpins or "dumbbells" (Germann et al., 1985; Wemmer and Benight, 1985; Erie et al., 1987, 1989; Benight et al., 1988; Ashley and Kushlan, 1991; Amaratunga et al., 1992; Doktycz et al., 1992; Paner et al., 1992). Because dumbbells denature in a monomolecular fashion and do not suffer from end effects, they are particularly useful in thermodynamic experiments (Erie et al., 1987, 1989). However, the synthesis of DNA dumbbells has in some cases proved to be quite challenging, thus limiting their utility (Erie et al., 1987). In addition, because the linkers are tethered to the terminal hydroxyl groups, some standard enzymatic manipulations such as end-labeling are not possible. of both loci to be bridged, as well as a chemistry

Methods for Cross-Linking Nucleic Acids

Contributed by Gary D. Glick

Current Protocols in Nucleic Acid Chemistry (2003) 5.7.1-5.7.13

Copyright $(\odot) 2003$ by John Wiley \& Sons, Inc.
5.7.1

Supplement 13 


\section{DESIGN OF AN IDEAL CROSS-LINK}

To develop a cross-linking approach that would be simple and general and would overcome the drawbacks of other methods, the author of this unit, and colleagues, chose eight important criteria that should be met (Table 5.7.1). It was clearly desirable to incorporate the cross-links by solid-phase synthesis to permit the modifications to be placed site-specifically and in close proximity within any given target sequence. Yet it was critical to find chemistry that would be specific and efficient for cross-link formation. Thiols were selected as the reactive functional groups because the mild redox chemistry used to form disulfide bonds from thiols is specific for sulfur. In addition, disulfide bonds are formed in high yield, often quantitatively, and are stable to a wide variety of solvents and reagents, yet are cleaved by reduction. Finally, the use of disulfide bonds to constrain macromolecular architecture has already been demonstrated in the peptide/protein literature (Clarke and Fersht, 1993, and references therein).

The next step in developing this chemistry was to select appropriate loci for modification. Because cross-links residing in either the major or minor groove could interfere with ligand recognition and hydration, cross-links were de-

Table 5.7.1 Requirements for an "Ideal" Nucleic Acid Cross-Link ${ }^{a}$

Requirements ${ }^{b}$
Increase the conformational
stability towards thermal-,
ionic-, pH-, and
concentration-induced
conformational changes
Form interstrand cross-link
site-specifically
Cross-link does not alter
native geometry

Grooves of the helices,
counter-ion binding, and
hydration must remain
unaltered
$3^{\prime}$ - and $5^{\prime}$-hydroxyl groups are
free to [32P]-end-label

Engineering

Terminal

Disulfide Bonds

Into DNA

5.7 .2

${ }^{a_{\text {From }} \text { Glick (1998). }}$

Rationale Potential solution

Prepared and isolated in large quantities

Reversible

Conformationally stable nucleic acids that are structurally homogeneous will facilitate their structural and thermodynamic analysis

Limits undesired adduct formation resulting in high yields of cross-linked product

Necessary if the structural aspects of cross-linked constructs are to be compared to their unmodified nucleic acid precursors

Allows for protein and ligand-nucleic acid interactions to be studied; maximizes (thermal) stability

Needed for wide range of biochemical assays (e.g., footprinting/sequencing)

Allows for high-resolution physical measurements such as NMR, DSC ${ }^{c}$, and X-ray diffraction studies

Assess structural and thermodynamic effects of after cross-linking

Flexible to a wide variety of nucleic acid structures base modification before and
Broad applicability will be necessary if this methodology is to be used uniformly in nucleic acids
Covalent interstand cross-links confer a large degree of conformational stability

Position functional groups proximal to one another on opposing strands of a helix Position cross-link within a sterically accessible space

Linkers at terminus of helix

Cross-link located on the base rather than terminal hydroxyl groups

Efficient cross-linking chemistry

Reversible disulfide cross-link

Position functional tether at any base or sugar site; cross-link within a sterically accessible space
${ }^{b}$ Relative to the unmodified nucleic acid.

${ }^{c}$ DSC, differential scanning calorimetry. 
signed at the terminus of the helical structure (Figure 5.7.1). To avoid using the $3^{\prime}$ - and 5'-hydroxyl groups, the terminal bases themselves were modified. This strategy exploited the fact that the terminal residues in duplexes have a reduced stability due to end effects, often referred to as "end fraying" (Patel and Hilbers, 1975). Therefore, altering the hydrogen bonding groups of the two opposing bases at the terminus of a duplex should not adversely affect helical stability, provided the thiol modifications do not disrupt base stacking. The free energy of a dT-dT mismatch located at the terminus of a duplex is $\sim 1.0 \mathrm{kcal} / \mathrm{mol}$ more stable than a dT-dT mismatch within a duplex (Aboul-ela et al., 1985; Freier et al., 1986). While this energetic penalty could be compensated for upon formation of the cross-link, it necessarily limits the overall (thermodynamic) stabilization the cross-link can provide.

When two thymidine residues directly oppose each other in duplex DNA, their N3 positions project toward the center of the helix and converge to $\sim 4.5 \AA$. Based on this observation, replacing the terminal bases on one or both ends of a duplex with N3-(alkylthiol)thymidine should be an ideal way to form interstrand disulfide cross-links. Molecular modeling studies suggest that disulfide cross-links can form when the terminal bases of a duplex are replaced with either N3-(methylthiol)thymidine or N3-(ethylthiol)thymidine. Since N3(methylthiol)thymidine is expected to decompose under the acidic conditions used to detritylate synthetic oligonucleotides, N3-(ethyl thiol)thymidine $\left(\mathrm{T}^{*}{ }_{\mathrm{SH}}\right)$ was chosen (Figure 5.7.1B).

The following sections describe key aspects pertaining to the synthesis, structure, dynamics, thermodynamic stability, and in vitro biological properties of these disulfide crosslinked DNA oligonucleotides. Two applications of this cross-linking chemistry are then discussed, as are recent experiments using different thiol-modified nucleosides to generate disulfide cross-links within RNA secondary and tertiary structure. Lastly, other methods that have been developed to incorporate disulfide cross-links into nucleic acids are briefly outlined.

\section{DISULFIDE CROSS-LINKED DNA DUPLEXES}

\section{Synthesis}

The properties of disulfide cross-linked DNA duplexes were explored by synthesizing analogs of two previously well-studied oligonucleotides. The first is a hairpin whose sequence is derived from the ColE1 cruciform
A

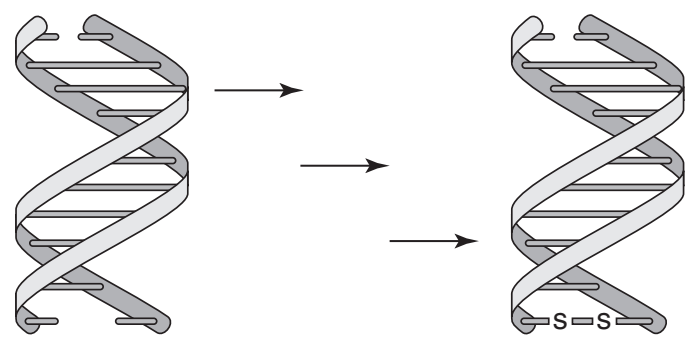

B

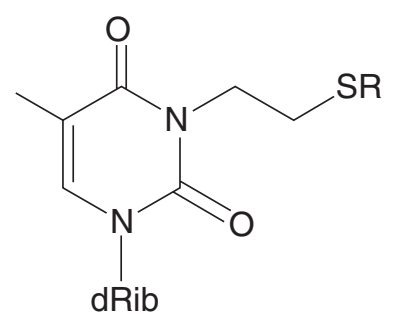

$$
\begin{aligned}
T^{\star}{ }_{\mathrm{SH}} ; \mathbf{R} & =\mathrm{H} \\
\mathrm{T}^{\star}{ }_{\mathrm{tBu}} ; \mathbf{R} & =\mathrm{StBu} \\
\mathrm{T}^{\star}{ }_{2} ; \mathbf{R} & =\succ_{2}
\end{aligned}
$$

Figure 5.7.1 ((A) Synthetic strategy. To place cross-links within a duplex requires synthesizing thiol-modified nucleosides, incorporating the modified bases within a target by solid-phase synthesis, removal of all protecting groups, and air oxidation to form the cross-link. (B) Chemical structure of modified thymidine and cross-link. During synthesis, the thiol group on $\mathrm{T}^{\star} \mathrm{SH}_{\mathrm{H}}$ is protected as a tert-butyl mixed disulfide $\left(\mathrm{T}_{\mathrm{tBu}}^{*}\right)$ and this protecting group is stable to all conditions of solid-phase synthesis, deprotection, and purification. The coupling efficiency of this base is indistinguishable from thymidine (>99\% per cycle). To place $\mathrm{T}_{\mathrm{tBu}}^{\star}$ at the $3^{\prime}$ terminus, the $3^{\prime}$-hydroxyl group is attached to controlled-pore glass. For $\mathrm{T}_{2}^{*}$, $\mathrm{R}$ is the disulfide cross-link. From Glick (1998).

Methods for Cross-Linking Nucleic Acids

\section{7 .3}

Supplement 13 

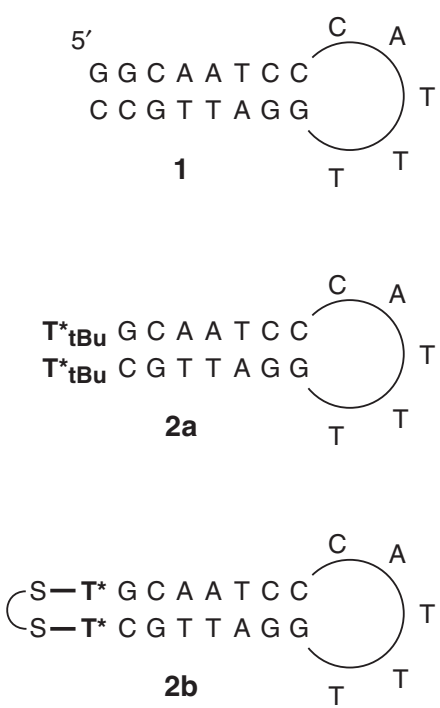

$5^{\prime}$

C G C G A A T T C G C G

GCGCT T A A G C G

3

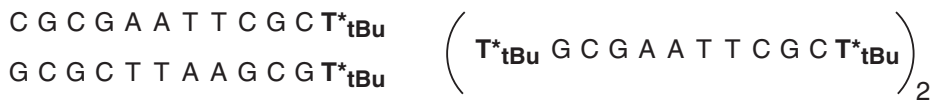

$4 a$

$5 a$

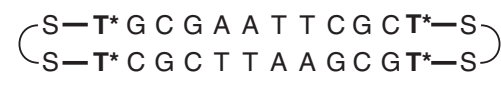

$5 b$

Figure 5.7.2 Duplex sequences and cross-linked analogs. From Glick (1998).

(S.1; Figure 5.7.2; Blatt et al., 1993), and the second is the Dickerson/Drew dodecamer (S.3; Wing et al., 1980). Synthesis of S.2a, S.4a, and S.5a is conducted using standard phosphoramidite chemistry using $N$-benzoyl- and $N$-isobutyryl-protected bases (Glick et al., 1992; Cain et al., 1995; Osborne et al., 1996). After deprotection and purification of each thiol-modified DNA by reversed-phase HPLC, the $t$-butyl mixed disulfide protecting groups on each sequence are cleaved with DTT ( 20 equiv per thiol, $\mathrm{pH} 8$, overnight at $4^{\circ} \mathrm{C}$ ). $\mathrm{Re}$ duced DNA samples are separated from the DTT by reversed-phase HPLC. Disulfide bond formation is performed by dissolving each sample (DNA concentration of $\sim 2 \mu \mathrm{M}$ ) in phosphate buffer ( $\mathrm{pH} 8.3)$ and stirring vigorously at $25^{\circ} \mathrm{C}$ with exposure to air. The reactions are usually complete in $8 \mathrm{hr}$, as determined by a negative Ellman's test. The cross-linked DNAs (S.2b, S.4b, and S.5b) are then isolated by either PAGE or HPLC, and incorporation of the modified bases is confirmed by enzymatic digestion. Importantly, disulfide bond formation is quantitative.

\section{Structural Studies}

The effects of introducing the thiol modifications were first assessed by comparing UV thermal denaturation profiles of S.2a to that of the corresponding wild-type sequence (S.1). The melting temperatures $\left(T_{\mathrm{m}}\right)$ values are within $1^{\circ} \mathrm{C}$ and the transitions are nearly superimposable, which suggests that the thiol linkers do not alter the stability or thermal denaturation pathways of the modified hairpin relative to its unmodified counterpart (Glick, 1991). These data also suggest that all of the stability conferred by the disulfide bond will be reflected in the cross-linked structure, since there is not a significant energetic penalty for replacing the terminal bases with the N3-thiol-modified thymidines. Introducing a disulfide cross-link in $\mathbf{S . 1}$ to generate $\mathbf{S . 2 b}$ increases the $T_{\mathrm{m}}$ from $65^{\circ}$ to $81^{\circ} \mathrm{C}$ in low-ionic-strength buffers. If the concentration of $\mathrm{Na}^{+}$is $>100 \mathrm{mM}$, the crosslinked hairpin denatures above $96^{\circ} \mathrm{C}$ and $T_{\mathrm{m}}$ values cannot be measured by UV spectroscopy (Glick, 1991).

To further investigate the structural effects of the disulfide modification, the solutionphase conformations of $\mathbf{S . 1}$ and $\mathbf{S . 2 b}$ were determined by NMR spectroscopy (Cain et al., 1995). The 8-bp-long stem of these sequences adopts a B-form helix, whereas the 5-bp-long single-stranded loop appears to be flexible and cannot be represented by a unique static conformation. NOESY cross-peak volumes, proton (both labile and non-labile) and phosphorus chemical shifts, as well as both homo- and heteronuclear coupling constants for the crosslinked hairpin are virtually identical to those measured for the unmodified sequence, even for the residues that are proximal to the crosslink. Thus, within the resolution of NMR spectroscopy, the two hairpins are structurally isomorphous. 
Similar to the results described above for the ColE1 hairpin, replacing one or both of the terminal $\mathrm{dG}-\mathrm{dC}$ base pairs in d(CGCGAATTCGCG $)_{2}$ with the modified thymidine does not significantly alter the thermal stability of these duplexes relative to the parent sequence (Osborne, 1996; Osborne et al., 1996). When the exchangeable and non-exchangeable protons and the ${ }^{31} \mathrm{P}$ resonances of S.5b are assigned and compared to those for the parent duplex, S.3, the spectra are virtually identical, which strongly indicates that these sequences also adopt very similar structures. (Because S.4b is not symmetric, a direct comparison with either $\mathbf{S . 3}$ or $\mathbf{S . 5 b}$ is not possible.) To provide further evidence of this point, 2-D NOESY spectra of $\mathbf{S . 5 b}$ as a function of mixing time can be measured and interproton distances obtained from initial build-up plots. These data for S.5b are nearly identical to those for the wild-type dodecamer. Based on these findings, along with the results of CD and nuclease-susceptibility experiments, these alkylthiol modifications, in either the disulfide cross-linked or reduced and protected forms, do not alter native structure (Osborne et al., 1996).

\section{Thermodynamic Measurements}

To elucidate the thermal and thermodynamic consequences of modifying and constraining DNA duplexes via the disulfide chemistry, differential scanning calorimetry (DSC) measurements can be conducted to characterize their thermally induced denaturation. DSC measurements on duplexes S.3 to S.5 (Osborne et al., 1996) represent the first use of calorimetry to investigate the thermodynamic consequences of constraining DNA with disulfide cross-links. The use of calorimetry is critical here because S.3 does not denature in a twostate fashion and cannot be analyzed using a van't Hoff treatment. Consistent with the NMR data, DSC data show that a large energetic penalty is not incurred by replacing the terminal base pair(s) with N3-(ethylthiol)thymidine: the $T_{\mathrm{m}}$ values of S.3, S.4a, and S.5a are within $11^{\circ} \mathrm{C}$ of each other. The overall free energies for S.3, S.4a, and S.5a are comparable; this similarity arises due to compensations in both enthalpy and entropy (Osborne, 1996).

The DSC measurements suggest that introducing cross-link(s) into $\mathbf{S . 3}$ results in two changes. First, constraining the dodecamer results in a significant increase in thermal stability. A single disulfide cross-link changes the molecularity of the complex from bimolecular to monomolecular (S.4b). However, a second disulfide cross-link (S.5b) results in a constrained conformation with a reduced entropy compared to S.4b. From the DSC data, the cross-link imparts $\sim 3 \mathrm{kcal} / \mathrm{mol}$ of stabilization. The change in entropy certainly reflects differences in the native and/or denatured states of $\mathbf{S . 5 b}$ compared to $\mathbf{S . 4 b}$. On first inspection, it would appear that the most likely source for the observed decrease in entropy is the denatured state, since the conformational freedom of the denatured state of $\mathbf{S . 5} \mathbf{b}$ is less than that of $\mathbf{S . 4 b}$. However, based on the data, differential entropic contributions from the native states, as well as influences from differential solvation in both the initial and the final states, cannot be excluded. Notwithstanding, the data show that the entropy value is responsible for the increase in thermal stability of $\mathbf{S . 5 b}$ relative to $\mathbf{S . 4 b}$. This is the first conformationally constrained nucleic acid system where the increase in melting temperature is predominantly due to a decrease in entropy (Erie et al., 1987, 1989).

\section{Dynamics Measurements}

The static structure of DNA can explain many aspects of its function and properties. However, a local base-pair opening is implicated in a number of important chemical, biological, and mechanical processes involving DNA (Frank-Kamenetskii, 1985; Ramstein and Lavery, 1988, 1990; Guéron and Leroy, 1995; Tari and Secco, 1995). Hence, defining the dynamics of base-pair opening is necessary to fully understand the physiochemical and biological properties of DNA. Although the opening kinetics for several different constructs have been measured, the opening kinetics for oligonucleotides constrained with cross-links (e.g., hairpin loops, glycol bridges) have not been reported (Gueron and Leroy, 1995). To address this, the base-pair lifetimes and apparent dissociation constants of $\mathbf{S . 1}$ and $\mathbf{S . 2 b}$ were measured (Cain and Glick, 1997). Comparison of the lifetimes and apparent dissociation constants for corresponding base-pairs of the two hairpins indicates that the cross-link neither increases the number of base-pairs involved in fraying nor alters the lifetime, dissociation constant, or the opened structure from which exchange occurs for the base-pairs that are not frayed. The cross-link does, however, stabilize the frayed penultimate base-pair of the stem duplex by increasing the closing rate of this base-pair. Significantly, the disulfide cross-link is more effective at preventing fraying than the 5-bp-long hairpin loop.
Methods for Cross-Linking Nucleic Acids

\section{$\mathbf{5 . 7 . 5}$}

Supplement 13 

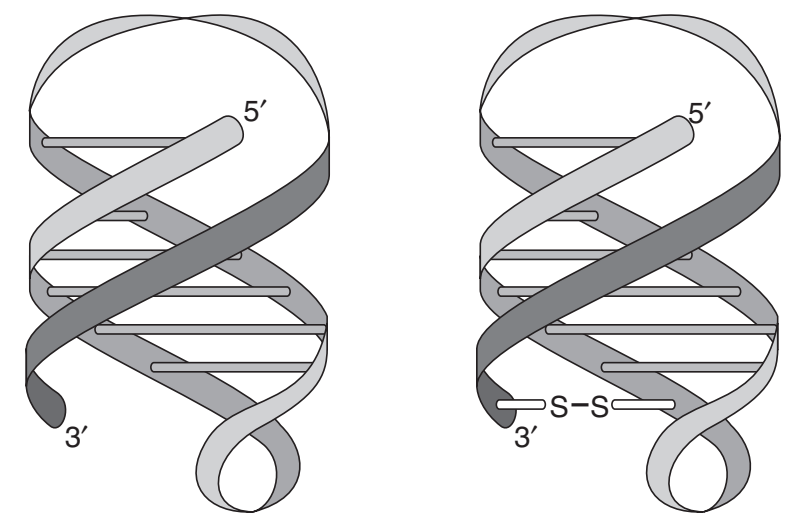

Figure 5.7.3 A triplex schematic. From Glick (1998).

\section{DISULFIDE CROSS-LINKED DNA TRIPLEXES}

\section{Design}

Folding of DNA triple helices based on the pyrimidine-purine-pyrimidine motif (py-pupy; dot $=$ Hoogsteen and dash $=$ Watson-Crick base-pairing) is affected by a number of factors including sequence length (i.e., the number of triplets formed), composition, the presence of base-pair mismatches, as well as solution conditions including $\mathrm{pH}$ and monovalent and divalent counterion concentrations (Moser and Dervan, 1987; Pilch et al., 1990; Kiessling et al., 1992; Plum et al., 1995). For example, a major factor in the stability of triplexes that contain $\mathrm{C}^{+} \cdot \mathrm{G}-\mathrm{C}$ triplets is the necessity for protonation of the N3 position of cytosine, which generally limits the stability of small triple helices of this type to $\mathrm{pH}$ below 7 (Callahan et al., 1991; Singleton and Dervan, 1992). The narrow $\mathrm{pH}$ range required for folding of these triplexes has hampered efforts to assess the physical properties of this motif under physiological conditions. Such data are clearly needed to design triplex sequences of higher affinity and specificity for diagnostic purposes in vitro and for use in vivo. While methods exist to stabilize py.pu-py triple helices, they rely on modifications that may alter the native triplex structure (Sun and Hélène, 1993; Thuong and Hélène, 1993). Therefore, it became important to determine if covalently locking the third strand of a triplex to the major groove through a structurally nonperturbing disulfide cross-link would afford constructs that are stable under physiological conditions. In other words, can the constraint provided by a disulfide cross-link alter the apparent $\mathrm{p} K_{\mathrm{a}}$ of a triplex?
This question was tested by using an intramolecular triplex rather than an intermolecular construct because the former should require only one cross-link to link the Hoogsteen strand to the Watson-Crick duplex (Figure 5.7.3; Goodwin et al., 1994; Osborne et al., 1997). To place a cross-link between the Hoogsteen and Watson-Crick strands, sites of chemical modification on the terminal bases must first be identified. In a T.T-A triplet, which is analogous to the base-pair substitution used to crosslink the terminus of duplexes (see Figure 5.7.1), the N3 position on the Hoogsteen thymidine and the C5 position of the Watson-Crick thymidine converge. Since the cross-link used for B-form duplexes is inappropriate here, a triplex cross-link was designed using a C5alkylthiol-modified thymidine $\left(\mathrm{C}_{3}^{5} \mathrm{~S}\right.$, where three is the number of atoms in the linker, including thiol) and N3-(ethylthiol)thymidine (Figure 5.7.4; Goodwin and Glick, 1993).

The optimal $\mathrm{pH}$ for air oxidation of thiols to form disulfide bonds is generally above the $\mathrm{p} K_{\mathrm{a}}$ of the thiol of interest ( 8.5). At the $\mathrm{p} K_{\mathrm{a}}$ of many thiols, triple helices containing $\mathrm{C}^{+} \cdot \mathrm{G}-\mathrm{C}$ base triplets are unfolded because the $\mathrm{N} 3$ position of cytosine is not protonated. To form a disulfide cross-link in a py-pu-py triplet, it is necessary to identify a sequence that (partly) folds into a triplex at a $\mathrm{pH}$ where cross-link formation readily proceeds. Recently, Häner and Dervan (1990) reported a 34-bp-long intramolecular triple helix that remains partially folded at up to $\mathrm{pH} 7.5$ at $24^{\circ} \mathrm{C}$ in a buffer containing $25 \mathrm{mM}$ $\mathrm{Mg}^{2+}$. In principle, therefore, a disulfide crosslink can form under the conditions needed to fold a suitably modified variant of this oligonucleotide (Figure 5.7.5). 


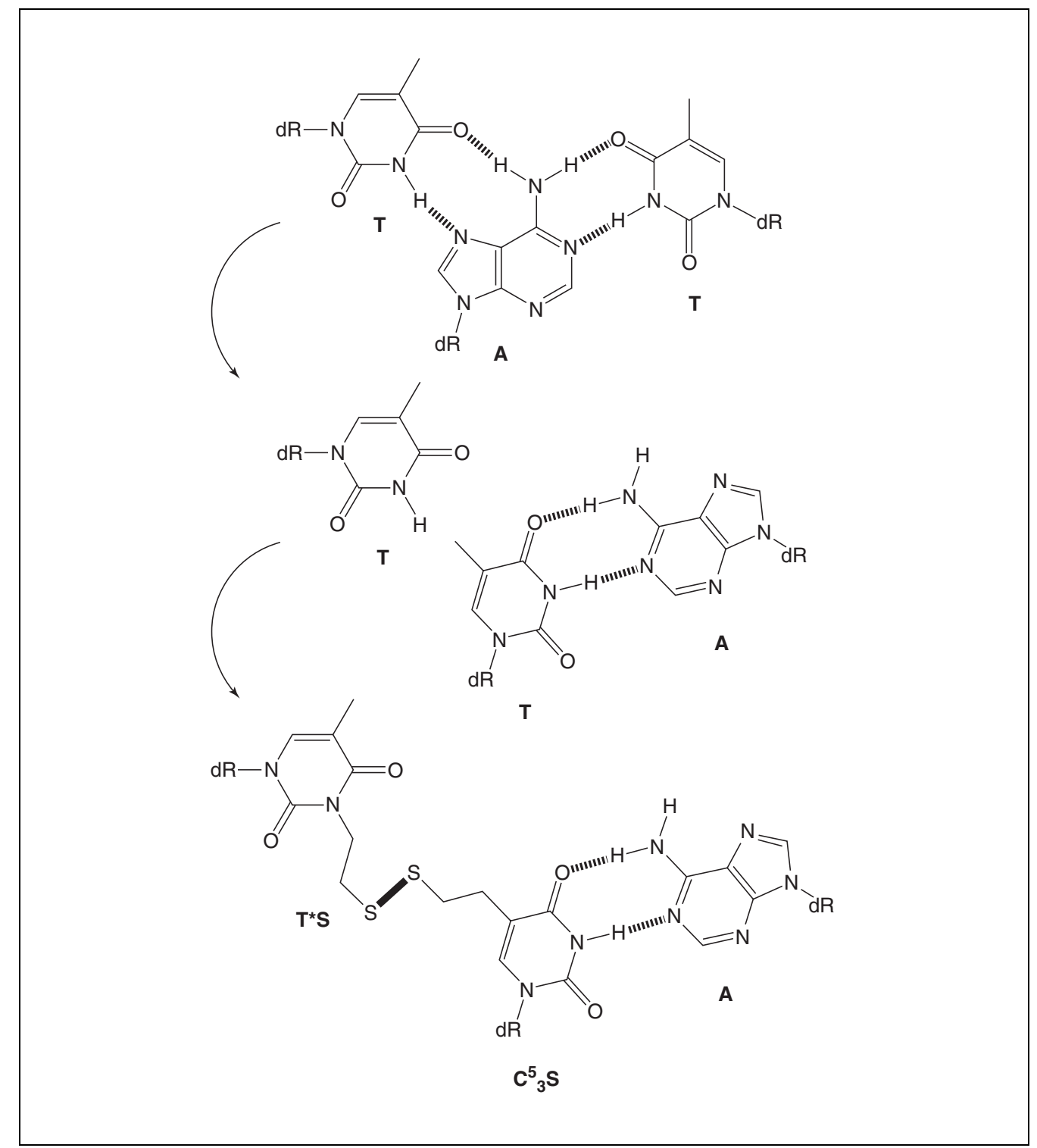

Figure 5.7.4 Chemical structure of triplex cross-link. From Glick (1998).

Preparation of triplex sequences containing $\mathrm{T}^{*}{ }_{\mathrm{tBu}}$ and $\mathrm{C}_{3}^{5} \mathrm{StBu}$ can be achieved via automated solid-phase DNA synthesis using standard protocols with an average stepwise coupling efficiency of $>98.5 \%$. Removal of the phosphate- and base-protecting groups followed by reversed-phase HPLC purification provides a $\sim 42 \%$ yield of S.7a (based on a $1-\mu \mathrm{mol}$ synthesis). To form the cross-link, the tert-butylthiol protecting groups are removed with DTT and the reduced sample is vigorously stirred at room temperature in PBS $(\mathrm{pH} 7.25,5$ $\mathrm{mM} \mathrm{MgCl} 2$ ) while exposed to air. After $24 \mathrm{hr}$, no starting material is observed by HPLC, and aliquots from the reaction mixtures test negative with Ellman's reagent. Cross-linked DNA is purified by reversed-phase HPLC to give S.7b in $\sim 28 \%$ isolated overall yield (based on a 1- $\mu$ mol synthesis; $\sim 75 \%$ yield from $\mathbf{S . 7 a}$ ). Enzymatic nucleoside composition analysis confirms incorporation of the modified bases and formation of the cross-link, and native and denaturing PAGE indicates that the structure formed is monomeric.

\section{Structural Studies}

When imino proton spectra of S.6 and S.7b are assigned using standard 2-D techniques under conditions that favor triplex formation (pH 6, $0.5 \mathrm{mM} \mathrm{Mg}^{2+}$ ), the imino proton spectra of both sequences are virtually identical, which indicates that the modifications do not disrupt native triplex structure (Osborne et al., 1997). When S.6 or S.7a is titrated from pH 6 to 8, dissociation of the third strand is observed. By contrast, the cross-linked triplex $\mathbf{S . 7 b}$ is con-
Methods for Cross-Linking Nucleic Acids 


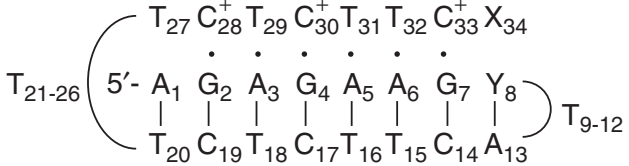

$$
\begin{aligned}
& \text { 6: } X, Y=T \\
& \left.\begin{array}{rlrl}
\text { 7a: } X & =\mathbf{T}^{*}{ }_{\text {tBu }} & \text { 7b: } X=T^{\star} \mathbf{S} \\
Y & =\mathbf{C}^{5}{ }_{3} \mathrm{~S}-\mathrm{StBu} & \mathrm{Y} & =\mathbf{C}^{5}{ }_{3} \mathbf{S}
\end{array}\right] \text { disulfide cross-link }
\end{aligned}
$$

Figure 5.7.5 Triplex sequence. From Glick (1998).

formationally stable over this $\mathrm{pH}$ range and only begins to unfold at $\mathrm{pH} \sim 9$. Moreover, $\mathbf{S . 7 b}$ is stable from $\mathrm{pH} 6$ to 8 in the absence of $\mathrm{Mg}^{2+}$. Because of line broadening above $5^{\circ} \mathrm{C}$, NMR cannot be used to study these triplexes. Therefore, CD spectroscopy is used. Monitoring the triplex CD signature band at $215 \mathrm{~nm}$ in the presence of $\mathrm{Mg}^{2+}, \mathbf{S . 7} \mathbf{b}$ does not begin to melt until $\sim 60^{\circ} \mathrm{C}$ at $\mathrm{pH} 6\left(30^{\circ} \mathrm{C}\right.$ for $\mathbf{S . 6}$ and $\left.\mathbf{S . 7 a}\right)$ and $40^{\circ} \mathrm{C}$ at $\mathrm{pH} 8\left(<20^{\circ} \mathrm{C}\right.$ for S.6 and S.7a). Constructing titration curves from the $\mathrm{CD}$ data reveals that the apparent $\mathrm{p} K_{\mathrm{a}}$ for $\mathbf{S . 7 b}$ is $\sim 8.6$, which is at least $1.5 \mathrm{p} K_{\mathrm{a}}$ units greater than $\mathbf{S . 6}$ and S.7a. These results clearly demonstrate that the presented cross-link can be used to stabilize higher-order DNA structures.

\section{Thermodynamic Measurements}

DSC measurements can be conducted to elucidate the thermodynamic consequences of modifying and constraining DNA triplexes with the disulfide chemistry (Völker et al., 1997). Cross-linked triplexes S.6 and S.7a melt in a biphasic manner above $\mathrm{pH} 6$, with the initial triplex-to-duplex transition (Hoogsteen strand release) occurring at lower temperatures than melting of the hairpin. In contrast, cross-linking increases the thermal stability of the Hoogsteen transition such that the triplex and hairpin duplex denature simultaneously. Model-independent thermodynamic data obtained by DSC reveal that the cross-link-induced increase in triplex thermal stability corresponds to a free energy stabilization of $\sim 3 \mathrm{kcal} / \mathrm{mol}$, with this stabilization being entirely entropic in origin. In other words, the cross-link is enthalpically neutral, but nevertheless induces a triplex stabilization of $3 \mathrm{kcal} / \mathrm{mol}$ due to a reduction in the entropy change associated with triplex melting. To deduce the origin(s) of this entropic impact, the $\mathrm{pH}$ and ionic strength dependence of the melting transitions were measured. From a comparison of the melting transitions at different $\mathrm{pH}$ values and ionic strengths, it can be estimated that 0.4 more protons are associated with the cross-linked triplex state than with the uncross-linked triplex, and 1.3 fewer counterions are released on melting the cross-linked triplex. Thus, the entropic stabilization is not solely a result of a reduction in conformational entropy.

\section{Dynamics Measurements}

At the start of this project, it was not known whether the base-pairs (Hoogsteen and Watson-Crick) that comprise triple-helical DNA open to any significant extent within a stable triplex. To address this question, the conformational dynamics of $\mathbf{S . 6}$ and $\mathbf{S . 7 b}$ were studied by 2-D exchange and NOE spectroscopy, and by measuring base-catalyzed imino-proton exchange rates (Cain and Glick, 1998). Under conditions that promote triplex formation $(\mathrm{pH}$ 6.0, $1^{\circ} \mathrm{C}$ ), S.6 and S.7b exhibit a small and identical degree of conformational heterogeneity. However, at higher temperatures $(\mathrm{pH} 6.0$, $37^{\circ} \mathrm{C}$ ), S.6 exhibits more extensive heterogeneity than S.7b. The exchange times for WatsonCrick imino protons are $\sim 1 \mathrm{hr}$ for both triplexes. However, the Hoogsteen base-pair lifetimes of S.6 could not be measured because this sequence is conformationally labile under the alkaline conditions necessary to conduct the exchange experiments.

Due to the extraordinary $\mathrm{pH}$ stability conferred by the cross-link, it is possible to measure the Hoogsteen lifetimes for $\mathbf{S . 7 b}$. The lifetimes of the these base-pairs range from $\sim 3$ to 370 msec. As for Watson-Crick base-pairs, the Hoogsteen lifetimes are highest at the central region within the triplex and taper off towards the termini, which is suggestive of imino ex- 

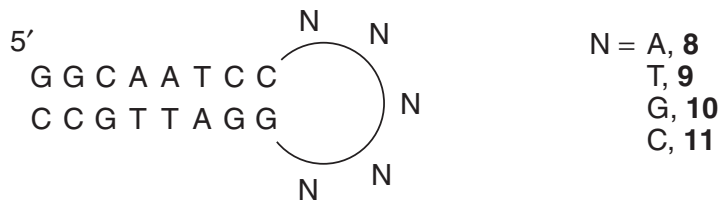

Figure 5.7.6 Hairpin ligands for the binding study. From Glick (1998).

change mechanisms involving end effects. In all cases, the lifetime of a Hoogsteen base-pair is shorter than that of the Watson-Crick basepair in the same triplet, which is consistent with the greater stability of Watson-Crick over Hoogsteen base-pairs. The rate of triplex-toduplex conversion for $\mathbf{S . 7 b}$ at $1^{\circ} \mathrm{C}$ and $\mathrm{pH} 6.9$ is low, with an upper bound of $3.2 \times 10^{-4} \mathrm{sec}^{-1}$. The imino protons of $\mathbf{S . 7 b}$ exchange slowly in PBS with exchange times as long as $1 \mathrm{hr}$, but the base-pair lifetimes are all $<3 \mathrm{~min}$, reflecting the fact that imino proton exchange is not opening-rate limited. Because the disulfide crosslink effectively prevents conformational heterogeneity associated with py-pu-py triple helices containing $\mathrm{C}^{+} \cdot \mathrm{G}-\mathrm{C}$ base triplets at neutral $\mathrm{pH}$, constructs possessing this modification can serve as model systems to examine the structural and thermodynamic aspects of triplex formation in vitro, and to develop sequences that bind DNA with higher affinity and specificity.

\section{APPLICATIONS}

Defining the biochemical, structural, dynamic, and thermodynamic impacts of constraining DNA with the disulfide chemistry described above opens the way for applying these cross-links in a host of different experiments. Uses of this chemistry include, among others, the joining of large pieces of DNA or RNA through disulfide bonds for the construction of nano-scale architectures, the design of redox-activated switches/devices, and the probing of secondary and tertiary structure. Below is a description of two representative applications of this chemistry. In the first, a disulfide bond was employed to stabilize or "trap" a DNA structure that would not be stable in its absence; in the second, a disulfide bond was used to report on conformational transitions in a protein binding study.

\section{Stabilizing Non-Ground-State Structures}

In the absence of disulfide cross-links, the molecules described in the above sections are themselves relatively stable with respect to conformational isomerization. In principle, however, it should also be possible to trap higherenergy non-ground-state structures with disulfide cross-links. Synthetic access to such constructs should be important in areas such as targeted drug delivery, protein-DNA recognition, and biophysical studies of alternate DNA geometries. The practicality of using disulfide cross-links in such endeavors can be demonstrated from the example of trapping the Dickerson/Drew dodecamer premelting intermediate with a disulfide cross-link.

Both S.3 and S.5a (Figure 5.7.2) thermally denature in a biphasic manner in buffers containing $10 \mathrm{mM} \mathrm{Na}^{+}$and $50 \mu \mathrm{M}$ DNA (Marky et al., 1983). The first transition defines melting of the duplex to a hairpin structure, while the second transition represents denaturation of the hairpin to a random coil. The premelting intermediate is cross-linked by air oxidation of the sulfhydryl groups in $\mathbf{S . 5 b}$ at $50^{\circ} \mathrm{C}$ in diluted solution with low-salt buffer (Glick et al., 1992). Unlike the stem-loop intermediate produced by initial melting of the parent dodecamer, the hairpin does not denature as a result of increasing temperature, $\mathrm{Na}^{+}$concentration, or DNA concentration (Glick et al., 1992; Wang et al., 1994).

To address the structural consequences of the cross-link, the solution-phase conformation of the hairpin was determined by NMR spectroscopy (Wang et al., 1994, 1995). The stem region of this hairpin forms a B-form DNA duplex with a helical rise of $3.5 \AA$ and a helical twist of $34.6^{\circ}$. The first three nucleotides in the loop stack over the $5^{\prime}$ end of the helix and are followed by a sharp turn at residue T8, which acts to close the loop. The conformation agrees with the "loop folding principle" advanced by Haasnoot, which predicts extension of the helix by 3 bases followed by a sharp bend in the loop (Haasnoot et al., 1986). The aromatic bases face into the major groove while the negatively charged backbone is in contact with solution.
Methods for Cross-Linking Nucleic Acids

\subsection{9}

Supplement 13 
Significantly, the cross-link does not alter the geometry of the stem duplex.

\section{Probes in Molecular Recognition Experiments}

DNA ligands often undergo conformational changes upon binding to proteins. In the absence of X-ray data, this "induced fit" can be difficult to observe. In principle, the constraint imposed by the disulfide bond should provide a way to investigate conformational changes in DNA structure that occur upon protein recognition. This point can be illustrated by the binding of monoclonal antibody BV04-01 to disulfide-cross-linked analogs of hairpins $\mathbf{S . 8}$ through S.11 (Figure 5.7.6; Stevens et al., 1993). This anti-DNA autoantibody was isolated from an autoimmune mouse that develops a syndrome related to human lupus, and BV0401 may be involved in the pathogenesis of this disorder in mice. BV04-01 binds only ssDNA, which can be modeled by the loop region of DNA hairpins (Stevens et al., 1993, and references therein).

If conformational reorganization of the hairpin ligands is required for binding, then BV0401 should possess a lower affinity for the crosslinked sequences, since the disulfide bond renders them resistant to structural changes. However, if preorganization is important for the formation of complexes, then the more rigid oligomers should bind with equal or greater affinity than the unmodified ligands. Measurements of BV04-01 binding to cross-linked hairpins reveals a nearly 100 -fold increase in $K_{\mathrm{d}}$ relative to S.8 through S.11. If the weaker affinity of BV04-01 for the cross-linked molecules results from the constraint imposed by the cross-link rather than as a result of a structural perturbation introduced by the alkylthiol linkers, then removing this constraint by reduction of the disulfide bond should afford a set of ligands that bind with roughly the same affinity as the unmodified hairpins. Indeed, BV04-01 recognition of the reduced hairpins is indistinguishable from binding to $\mathbf{S . 8}$ through $\mathbf{S . 1 1}$. DNA footprinting experiments show that, upon binding, residues within the duplex are recognized by single-strand-specific reagents, which provides further evidence that the stem duplex of the hairpin ligands is partially denatured (Swanson et al., 1994).

\section{SYNTHESIS OF DISULFIDE CROSS-LINKED DNAs USING CONVERTIBLE NUCLEOSIDES}

The chemistry developed and advanced by Verdine and co-workers stands as the best method to place cross-links within the helical regions of DNA (Ferentz et al., 1991, 1993). Using convertible nucleoside chemistry, they showed that placing $N^{6}$-thioalkyl derivatives of 2 -deoxyadenosine in consecutive base pairs on opposite strands of a duplex afforded disulfide cross-links upon air oxidation. These crosslinks reside in the major groove and impart increased stability with minimal distortion of native DNA geometry. This chemistry was extended several years latter to the synthesis of minor groove cross-links using modified $\mathrm{dC}$ residues. These major and minor groove crosslinks have been used with excellent effect in a variety of ways, such as studying protein-DNA interactions (Erlanson et al., 1993, 1997), stabilizing intrinsically bent DNA (Wolfe and Verdine, 1993), inducing torsional stress in short oligonucleotides (Wolfe et al., 1995), and cross-linking Z-DNA (Wolfe and Verdine, 1993).

\section{Literature Cited}

Aboul-ela, F., Koh, D., Tinoco, I. Jr., and Martin, F.H. 1985. Base-base mismatches. Thermodynamics of double-helix formation for $\mathrm{dCA}_{3} \mathrm{XA}_{3} \mathrm{G}+\mathrm{dCT}_{3} \mathrm{YT}_{3} \mathrm{G}(\mathrm{X}, \mathrm{Y}=\mathrm{A}, \mathrm{C}, \mathrm{G}, \mathrm{T})$. Nucl. Acids Res. 13:4811-4824.

Amaratunga, M., Snowden-Ifft, E., Wemmer, D.E., and Benight, A.S. 1992. Studies of DNA dumbbells. II. Construction and characterization of DNA dumbbells with a 16 base-pair duplex stem and $\mathrm{Tn}$ end loops $(\mathrm{n}=2,3,4,6,8,10,14)$. Biopolymers 32:865-879.

Ashley, G.W. and Kushlan, D.M. 1991. Chemical synthesis of oligodeoxynucleotide dumbbells. Biochemistry 30:2927-2933.

Baldwin, R.L. 1971. Experimental tests of the theory of deoxyribonucleic acid melting with d(TA) oligomers. Acc. Chem. Res. 4:265-272.

Bannwarth, W., Dorn, A., Iaiza, P., and Pannekouke, X. 1994. Short optimally capped duplex DNA as a conformationally restricted analog of B-DNA. Helv. Chim. Acta 77:182-193.

Benight, A.S., Schurr, J.M., Flynn, P.F., Reid, B.R., and Wemmer, D.E. 1988. Melting of a self-complementary minicircle. Comparison of optical melting theory with exchange broadening of the nuclear magnetic resonance spectrum. J. Mol. Biol. 200:377-399.

Blatt, N.B., Osborne, S.E., Cain, R.J., and Glick, G.D. 1993. Conformational studies from the ColE1 cruciform. Biochimie 75:433.
Engineering

Terminal

Disulfide Bonds

Into DNA

5.7.10 
Boger, D.L., Munk, S.A., and Ishizaki, T. 1991. (+)-CC-1065 DNA alkylation: Observation of an unexpected relationship between cyclopropane electrophile reactivity and the intensity of DNA alkylation. J. Am. Chem. Soc. 113:2779-2780.

Borowy-Borowski, H., Lipman, R., and Tomasz, M. 1990. Recognition between mitomycin $\mathrm{C}$ and specific DNA sequences for cross-link formation. Biochemistry 29:2999-3006.

Breslauer, K.J. 1986. Thermodynamics of nucleic acids. In Thermodynamic Data for Biochemistry and Biotechnology (H.J. Hinz, ed.) pp 402-427. Springer-Verlag, New York.

Breslauer, K.J., Frank, R., Blöcker, H., and Marky, L.A. 1986. Predicting DNA duplex stability from the base sequence. Proc. Natl. Acad. Sci. U.S.A. 83:3746-3750.

Cain, R.J. and Glick, G.D. 1997. The effect of cross-links on the conformational dynamics of duplex DNA. Nucl. Acids Res. 25:836-842.

Cain, R.J. and Glick, G.D. 1998. Use of cross-links to study the conformational dynamics of triplex DNA. Biochemistry 37:1456-1464.

Cain, R.J., Zuiderweg, E.R.P., and Glick, G.D. 1995. Solution structure of a DNA hairpin and its disulfide cross-linked analog. Nucl. Acids Res. 23:2153-2160.

Callahan, D.E., Trapane, T.L., Miller, P.S., Ts'o, P.O.P., and Kan, L.-S. 1991. Comparative circular dichroism and fluorescence studies of oligodeoxyribonucleotide and oligodeoxyribonucleoside methylphosphonate pyrimidine strands in duplex and triplex formation. Biochemistry 30:1650-1655.

Catalano, C.E. and Benkovic, S.J. 1989. Inactivation of DNA polymerase I (Klenow fragment) by adenosine 2',3'-epoxide 5'-triphosphate: Evidence for the formation of a tight-binding inhibitor. Biochemistry 28:4374-4382.

Clarke, J. and Fersht, A.R. 1993. Engineered disulfide bonds as probes of the folding pathway of barnase: Increasing the stability of proteins against the rate of denaturation. Biochemistry 32:4322-4329.

Cowart, M. and Benkovic, S.J. 1991. A novel combined chemical-enzymic synthesis of crosslinked DNA using a nucleoside triphosphate analog. Biochemistry 30:788-796.

Cowart, M., Gibson, K.J., Allen, D.J., and Benkovic, S.J. 1989. DNA substrate structural requirements for the exonuclease and polymerase activities of prokaryotic and phage DNA polymerases. Biochemistry 28:1975-1983.

Doktycz, M.J., Goldstein, R.F., Paner, T.M., Gallo, F.J., and Benight, A.S. 1992. Studies of DNA dumbbells. I. Melting curves of 17 DNA dumbbells with different duplex stem sequences linked by T4 endloops: Evaluation of the nearest-neighbor stacking interactions in DNA. Biopolymers 32:849-864.
Durand, M., Chevrie, K., Chassignol, M., Thuong, N.T., and Maurizot, J.C. 1990. Circular dichroism studies of an oligodeoxyribonucleotide containing a hairpin loop made of a hexaethylene glycol chain: Conformation and stability. Nucl. Acids Res. 18:6353-6359.

Elson, E.L., Scheffler, I.E., and Baldwin, R.L. 1970. Helix formation by d(TA) oligomers. 3. Electrostatic effects. J. Mol. Biol. 54:401-415.

Erie, D.A., Sinha, N., Olson, W.K., Jones, R.A., and Breslauer, K.J. 1987. A dumbbell-shaped double-hairpin structure of DNA: A thermodynamic investigation. Biochemistry 26:7150-7159.

Erie, D.A., Sinha, N.K., Olson, W.K., Jones, R.A., and Breslauer, K.J. 1989. Melting behavior of covalently closed, single-stranded, circular DNA. Biochemistry 28:268-273.

Erlanson, D.A., Chen, L., and Verdine, G.L. 1993. DNA methylation through a locally unpaired intermediate. J. Am. Chem. Soc. 115:1258312584.

Erlanson, D.A., Wolfe, S.A., Chen, L., and Verdine, G.L. 1997. Selective base-pair destabilization enhances binding of a DNA methyltransferase. Tetrahedron 53:12041-12056.

Ferentz, A.E. and Verdine, G.L. 1991. Disulfidecross-linked oligonucleotides. J. Am. Chem. Soc. 113:4000-4002.

Ferentz, A.E., Keating, T.A., and Verdine, G.L. 1993. Synthesis and characterization of disulfide cross-linked oligonucleotides. J. Am. Chem. Soc. 115:9006-9014.

Frank-Kamenetskii, M.D. 1985. Flunctuational motility of DNA. In Structure \& Motion: Membranes, Nucleic Acids, and Proteins (E. Clementi, G. Corongiu, M.H. Sarma, and R.H. Sarma, eds.) pp. 417-432. Adenine Press, Guilderland, N.Y.

Freier, S.M., Kierzek, R., Caruthers, M.H., Neilson, T., and Turner, D.H. 1986. Free energy contributions of G.U and other terminal mismatches to helix stability. Biochemistry 25:3209-3213.

Gao, H., Chidambaram, N., Chen, B.C., Pelham, D.E., Patel, R., Yang, M., Zhou, L., Cook, A., and Cohen, J.S. 1994. Double-stranded cyclic oligonucleotides with non-nucleotide bridges. Bioconjugate Chem. 5:445-453.

Gao, H., Yang, M., and Cook, A.F. 1995. Stabilization of double-stranded oligonucleotides using backbone-linked disulfide bridges. Nucl. Acids Res. 23:285-292.

Germann, M.W., Schoenwaelder, K.-H., and van de Sande, J.H. 1985. Right- and left-handed (Z) helical conformations of the hairpin $\mathrm{d}(\mathrm{C}$ $\mathrm{G})_{5} \mathrm{~T}_{4}(\mathrm{C}-\mathrm{G})_{5}$ monomer and dimer. Biochemistry 24:5698-5702.

Glick, G.D. 1991. Synthesis of a conformationally restricted DNA hairpin. J. Org. Chem. 56:67466747.

Glick, G.D., 1998. Design, synthesis, and analysis of conformationally constrained nucleic acids. Biopolymers 48:83-96.
Methods for Cross-Linking Nucleic Acids

\subsubsection{1}

Supplement 13 
Glick, G.D., Osborne, S.E., Knitt, D.S., and Marino, J.P. Jr. 1992. Trapping and isolation of an alternate DNA conformation. J. Am. Chem. Soc. 114:5447-5448.

Goodwin, J.T. and Glick, G.D. 1993. Incorporation of alkylthiol chains at C-5 of deoxyuridine Tetrahedron Lett. 34:5549-5552.

Goodwin, J.T., Osborne, S.E., Swanson, P.C., and Glick, G.D. 1994. Synthesis of a disulfide crosslinked DNA triple helix. Tetrahedron Lett. 35:4527-4531.

Guéron, M. and Leroy, J.L. 1995. Studies of base pair kinetics by NMR measurement of proton exchange. Methods Enzymol. 261:383-413.

Haasnoot, C.A.G., Hilbers, C.W., van der Marel, G.A., van Boom, J.H., Singh, U.C., Pattabiraman, N., and Kollman, P.A. 1986. On loop folding in nucleic acid hairpin-type structures. $J$. Biomol. Struct. Dyn. 3:843-857.

Häner, R. and Dervan, P.B. 1990. Single-stranded DNA triple-helix formation. Biochemistry 29:9761-9765.

Kiessling, L.L., Griffin, L.C., and Dervan, P.B. 1992. Flanking sequence effects within the pyrimidine triple-helix motif characterized by affinity cleaving. Biochemistry 31:2829-2834.

Kirchner, J.J. and Hopkins, P.B. 1991. Nitrous acid cross-links duplex DNA fragments through deoxyguanosine residues at the sequence $5^{\prime}$-CG. $J$. Am. Chem. Soc. 113:4681-4682.

Kirchner, J.J., Sigurdsson, S.T., and Hopkins, P.B. 1992. Interstrand cross-linking of duplex DNA by nitrous acid: Covalent structure of the dG-todG cross-link at the sequence 5'-CG. J. Am. Chem. Soc. 114:4021-4027.

Kool, E.T. 1991. Molecular recognition by circular oligonucleotides: Increasing the selectivity of DNA binding. J. Am. Chem. Soc. 113:62656266.

Lemaire, M.-A., Schwartz, A., Rahmouni, A.R., and Leng, M. 1991. Interstrand cross-links are preferentially formed at the $\mathrm{d}(\mathrm{GC})$ sites in the reaction between cis-diamminedichloroplatinum(II) and DNA. Proc. Natl. Acad. Sci. U.S.A. 88:19821985.

MacGregor, R.B. Jr. 1996. Chain length and oligonucleotide stability at high pressure. Biopolymers 38:321-327.

Marky, L.A., Blumenfeld, K.S., Kozlowski, S., and Breslauer, K.J. 1983. Salt-dependent conformational transitions in the self-complementary deoxydodecanucleotide d(CGCAATTCGCG): Evidence for hairpin formation. Biopolymers 22:1247-1257.

Millard, J.T., Raucher, S., and Hopkins, P.B. 1990. Mechlorethamine cross-links deoxyguanosine residues at $5^{\prime}$-GNC sequences in duplex DNA fragments. J. Am. Chem. Soc. 112:2459-2460.
Millard, J.T., Weidner, M.F., Kirchner, J.J., Ribeiro, S., and Hopkins, P.B. 1991. Sequence preferences of DNA interstrand crosslinking agents: Quantitation of interstrand crosslink locations in DNA duplex fragments containing multiple crosslinkable sites. Nucl. Acids Res. 19:18851891.

Moser, H.E. and Dervan, P.B. 1987. Sequence-specific cleavage of double helical DNA by triple helix formation. Science 238:645-650.

Olmsted, M.C., Anderson, C.F., and Record, M.T. Jr. 1991. Importance of oligoelectrolyte end effects for the thermodynamics of conformational transitions of nucleic acid oligomers: A grand canonical Monte Carlo analysis. Biopolymers 31:1593-1604.

Osborne, S.E. 1996. Ph.D. Thesis, University of Michigan.

Osborne, S.E., Völker, J., Stevens, S.Y., Breslauer, K.J., and Glick, G.D. 1996. Design, synthesis, and analysis of disulfide cross-linked DNA duplexes. J. Am. Chem. Soc. 118:11993-12003.

Osborne, S.E., Cain, R.J., and Glick, G.D. 1997. Structure and dynamics of disulfide cross-linked DNA triple helices. J. Am. Chem. Soc. 119:11711182.

Paner, T.M., Amaratunga, M., and Benight, A.S. 1992. Studies of DNA dumbbells. III. Theoretical analysis of optical melting curves of dumbbells with a 16 base-pair duplex stem and Tn end loops $(\mathrm{n}=2,3,4,6,8,10,14)$. Biopolymers 32:881-892.

Patel, D.J. and Hilbers, C.W. 1975. Proton nuclear magnetic resonance investigations in doublestranded dApTpGpCpApT in aqueous solution. Biochemistry 14:2651-2656.

Pilch, D.S., Levenson, C., and Shafer, R.H. 1990. Structural analysis of the $\mathrm{d}(\mathrm{A})_{10} .2(\mathrm{dT})_{10}$ triple helix. Proc. Natl. Acad. Sci. U.S.A. 87:19421946.

Pinto, A.L. and Lippard, S. 1985. Binding of the antitumor drug cis-diamminedichloroplatinum(II) (cisplatin) to DNA. Biochem. Biophys. Acta 780:167-180.

Plum, G.E., Pilch, D.S., Singleton, S.F., and Breslauer, K.J. 1995. Nucleic acid hybridization: Triplex stability and energetics. Annu. Rev. Biophys. Biomol. Struct. 24:319-350.

Ramstein, J. and Lavery, R. 1988. Energetic coupling between DNA bending and base pair opening. Proc. Natl. Acad. Sci. U.S.A. 85:7231-7235.

Ramstein, J. and Lavery, R. 1990. Base pair opening pathways in B-DNA. J. Biomol. Struct. Dyn. 7:915-933

Record, M.T. Jr. and Lohman, T.M. 1978. Semi-empirical extension of polyelectrolyte theory to treatment of oligoelectrolytes-application to oligonucleotide helix-coil transitions. Biopolymers 17:159-166.

Rumney, S. IV and Kool, E.T. 1992. DNA recognition by hybrid oligoether-oligodeoxynucleotide macrocycles. Angew. Chem. Int. Ed. Engl. 31:1617-1619. 
Salunkhe, M., Wu, T., and Lestinger, R.L. 1992. Control of folding and binding of oligonucleotides by use of a nonnucleotide linker. J. Am. Chem. Soc. 114:8768-8772.

SantaLucia, J. Jr., Allawi, H.T., and Seneviratne, P.A. 1996. Improved nearest-neighbor parameters for predicting DNA duplex stability. Biochemistry 35:3555-3562.

Scheffler, I.E., Elson, E.L., and Baldwin, R.L. 1970. Helix formation by d(TA) oligomers. II. Analysis of the helix-coil transitions of linear and circular oligomers. J. Mol. Biol. 48:145-171.

Sherman, S.E., Gibson, D., Wang, A.H., and Lippard, S.J. 1985. X-ray structure of the major adduct of the anticancer drug cisplatin with DNA: cis-[Pt(NH3)2] (d(pGpG)). Science 230:412-417.

Sigurdsson, S.T., Rink, S.M., and Hopkins, P.B. 1993. Affinity crosslinking of duplex DNA by a pyrrole-oligopeptide conjugate. J. Am. Chem. Soc. 115:12633-12634.

Singleton, S.F. and Dervan, P.B. 1992. Influence of $\mathrm{pH}$ on the equilibrium association constants for oligodeoxyribonucleotide-directed triple helix formation at single DNA sites. Biochemistry 31:10995-11003.

Stevens, S.Y., Swanson, P.C., Voss, E.W. Jr., and Glick, G.D. 1993. Evidence for induced fit in antibody.DNA complexes. J. Am. Chem. Soc. 115:1585-1586.

Sun, J.-S. and Hélène, C. 1993. Oligonucleotide-directed triple-helix formation. Curr. Opin. Struct. Biol. 3:345-356.

Swanson, P.C., Cooper, B.C., and Glick, G.D. 1994. High-resolution epitope mapping of an antiDNA autoantibody using model DNA ligands. $J$. Immunol. 152:2601-2612.

Tari, L.W. and Secco, A.S. 1995. Base-pair opening and spermine binding-B-DNA features displayed in the crystal structure of a gal operon fragment: Implications for protein-DNA recognition. Nucl. Acids Res. 23:2065-2073.

Teng, S.P., Woodson, S.A., and Crothers, D.M. 1989. DNA sequence specificity of mitomycin cross-linking. Biochemistry 28:3901-3907.

Thuong, N.T. and Hélène, C. 1993. Sequence-specific recognition and modification of doublehelical DNA by oligonucleotides. Angew. Chem. Int. Ed. Engl. 32:666-690.
Tomasz, M., Lipman, R., Chowdary, D., Pawlak, J., Verdine, G.L., and Nakanishi, K. 1987. Isolation and structure of a covalent cross-link adduct between mitomycin C and DNA. Science 235:1204-1208.

Völker, J., Osborne, S.E., Glick, G.D., and Breslauer, K.J. 1997. Thermodynamic properties of a conformationally constrained intramolecular DNA triple helix. Biochemistry 36:756-767.

Wang, H., Osborne, S.E., Zuiderweg, E.R.P., and Glick, G.D. 1994. Three-dimensional structure of a disulfide-stabilized non-ground-state DNA hairpin. J. Am. Chem. Soc. 116:5021-5022.

Wang, H., Zuiderweg, E.R.P., and Glick, G.D. 1995. Solution structure of a disulfide cross-linked DNA hairpin. J. Am. Chem. Soc. 117:2981-2991.

Webb, T.R. and Matteucci, M.D. 1986. Sequencespecific cross-linking of deoxyoligonucleotides via hybridization-triggered alkylation. J. Am. Chem. Soc. 108:2764-2765.

Wemmer, D.E. and Benight, A.S. 1985. Preparation and melting of single-strand circular DNA loops. Nucl. Acids Res. 13:8611-8621.

Williams, D.J. and Hall, K.B. 1996. Thermodynamic comparison of the salt dependence of natural RNA hairpins and RNA hairpins with non-nucleotide spacers. Biochemistry 35:1466514670.

Willis, M.C., Hicke, B.J., Uhlenbeck, O.C., Cech, T.R., and Koch, T.H. 1993. Photocrosslinking of 5-iodouracil-substituted RNA and DNA to proteins. Science 262:1255-1257.

Wing, R., Drew, H., Takano, T., Broka, C., Tanaka, S., Itakura, K., and Dickerson, R.E. 1980. Crystal structure analysis of a complete turn of BDNA. Nature 287:755-758.

Wolfe, S.A. and Verdine, G.L. 1993. Ratcheting torsional stress in duplex DNA. J. Am. Chem. Soc. 115:12585-12586.

Wolfe, S.A., Ferentz, A.E., Grantcharova, V., Churchill, M.E.A., and Verdine, G.L. 1995. Modifying the helical structure of DNA by design: Recruitment of an architecture-specific protein to an enforced DNA bend. Chem. Biol. 2:213-221.

Contributed by Gary D. Glick

University of Michigan

Ann Arbor, Michigan

The author wishes to thank the talented group of associates in his laboratory who have so skillfully conducted the research described in this unit. He also thanks K.J. Breslauer, Jens Völker, and E.R.P. Zuiderweg, whose expertise and insight into nucleic acids has formed the basis for outstanding collaborations. The worked described here was supported by NIH Grants GM-52831 and GM43861.

Methods for Cross-Linking Nucleic Acids

\section{7 .13}

Supplement 13 